\title{
The massive binary HD 152218 revisited: a new colliding wind system in NGC $6231^{\star}$
}

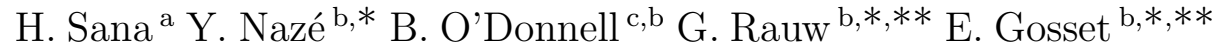 \\ ${ }^{a}$ European Southern Observatory, Alonso de Cordova 310\%, Vitacura, Santiago 19, Chile \\ ${ }^{\mathrm{b}}$ Institut d'Astrophysique et de Géophysique, Liège University, Allée du 6 Août 17, Bat. B5c, B-4000 Liège, Belgium \\ ${ }^{\mathrm{c}}$ Dept. of Physics 83 Astronomy, University College London, Gower Street, London WC1E 6BT, UK.
}

\section{Abstract}

We present the results of an optical and X-ray monitoring campaign on the short-period massive SB2 binary HD 152218 . Combining our HiRes spectroscopic data with previous observations, we unveil the contradictions between the published orbital solutions. In particular, we solve the aliasing on the period and derive a value close to $5.604 \mathrm{~d}$. Our eccentricity $e=0.259 \pm 0.006$ is slightly lower than previously admitted. We show that HD 152218 is probably undergoing a relatively rapid apsidal motion of about $3^{\circ} \mathrm{yr}^{-1}$ and we confirm the O9IV+O9.7V classification. We derive minimal masses of $15.82 \pm 0.26 \mathrm{M}_{\odot}$ and $12.00 \pm 0.19 \mathrm{M}_{\odot}$ and constrain the radius of the components to $R_{1}=10.3 \pm 1.3 \mathrm{R}_{\odot}$ and $R_{2}=7.8 \pm 1.7 \mathrm{R} \odot$. We also report the results of a XMM-Newton monitoring of the HD 152218 X-ray emission throughout its orbital motion. The averaged X-ray spectrum is relatively soft and it is well reproduced by a $2-\mathrm{T}$ optically thin thermal plasma model with component temperatures about 0.3 and $0.7 \mathrm{keV}$. The system presents an increase of its X-ray flux by about $30 \%$ near apastron compared to periastron, which is interpreted as the signature of an ongoing wind-wind interaction process occurring within the wind acceleration region.

Key words: stars: individual: HD 152218, stars: binaries: spectroscopic, stars: early-type, X-rays: individual: HD 152218

PACS: 97.10.Me, 97.20.Ec, 97.80.Fk, 98.70.Qy

\section{Introduction}

Early-type stars of spectral type $\mathrm{O}$ are characterized by strong stellar winds. Though not as extreme as those of their evolved descendants, the Wolf-Rayet stars, these winds combine terminal velocities of a few thousand $\mathrm{kms}^{-1}$ and important mass-loss rates (about $10^{-7}-10^{-5} \mathrm{M}_{\odot} \mathrm{yr}^{-1}$ ) that significantly affect both the surroundings of the star and its evolution. In a binary system, it is expected that the winds from the two stars collide, producing a density enhanced region known as the wind interaction zone. The shock-heated plasma within

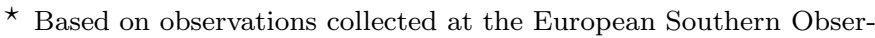
vatory (La Silla, Chile), at the Cerro Tololo Inter-American Observatory (Cerro Tololo, Chile) and with XMM-Newton, an ESA Science Mission with instruments and contributions directly funded by ESA Member States and the USA (NASA).

* FNRS, Belgium

**Visiting Astronomer, CTIO, National Optical Astronomy Observatories (NOAO). NOAO is operated by the Association of Universities for Research in Astronomy, Inc. under contract with the National Science Foundation.
}

this zone is expected to produce an additional contribution to the X-ray emission from the early-type system and, indeed, the early-type binaries are known to be statistically X-ray overluminous compared to single stars of the same spectral type (Chlebowski \& Garmany, 1991). This extra $\mathrm{X}$-ray emission might display phase-locked modulations, either due to a change of the absorption properties along the line of sight, or to a modulation of the shock strength due e.g. to a varying separation between the components in an eccentric binary. The properties of the shock thus strongly depend both on the geometry of the binary system and on the characteristics of the individual winds. However these properties often cruelly lack accurate constraints. In this framework, our team is involved in a long-standing effort to study early-type stars in a number of open clusters.

The present work focuses on the short-period binary HD152218, located in NGC 6231 at the core of the Sco OB 1 association. This star is a long known SB2 system (Struve, 1944). Previous papers (Hill et al.,, 1974; Levato \& Morrell, 1983; Stickland et al., 1997; García \& Mermilliod, 2001) however reported conflicting results and we thus decided to re-appraise the orbital properties of this apparently well- 
Table 1

Journal of the spectroscopic observations of HD 152218. Column 1 lists the Heliocentric Julian Date (in format HJD-2 450 000) at mid-

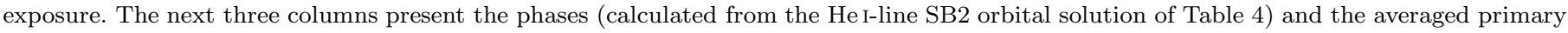

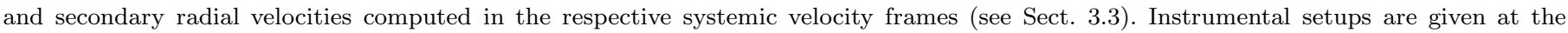
bottom of the table.

\begin{tabular}{|c|c|c|c|c|c|}
\hline \multicolumn{3}{|c|}{ HJD $\phi_{\mathrm{HeI}} \overline{R V_{\lambda, 1}-\gamma_{\lambda, 1}} \overline{R V_{\lambda, 2}-\gamma_{\lambda, 2}}$} & \multicolumn{3}{|c|}{ HJD $\phi_{\mathrm{HeI}} \overline{R V_{\lambda, 1}-\gamma_{\lambda, 1}} \overline{R V_{\lambda, 2}-\gamma_{\lambda, 2}}$} \\
\hline $621.673^{a} 0.406$ & -25.00 & -29.31 & $1302.901^{c} 0.968$ & -2.09 & 0.46 \\
\hline $622.642^{a} 0.579$ & 63.69 & -133.73 & $1304.902^{c} 0.325$ & -82.39 & 134.85 \\
\hline $622.755^{a} 0.599$ & 80.30 & -123.20 & $1323.853^{c} \quad 0.707$ & 132.51 & -175.86 \\
\hline $623.648^{a} 0.759$ & 151.20 & -200.79 & $1327.796^{c} 0.411$ & -11.80 & -9.25 \\
\hline $623.762^{a} 0.779$ & 163.30 & -188.40 & $1328.905^{b} 0.609$ & 82.27 & -124.90 \\
\hline $624.590^{a} 0.927$ & 74.35 & -116.83 & $1329.855^{b} 0.778$ & 147.17 & -199.78 \\
\hline $624.722^{a} 0.950$ & 13.36 & 9.05 & $1331.882^{b} 0.140$ & -182.39 & 213.02 \\
\hline $625.626^{a} 0.112$ & -158.93 & 209.21 & $1669.812^{c} 0.442$ & -6.42 & -3.87 \\
\hline $625.734^{a} 0.131$ & -171.70 & 233.10 & $1670.804^{c} 0.619$ & 96.65 & -114.87 \\
\hline $626.591^{a} 0.284$ & -104.57 & 166.73 & $1670.918^{c} 0.639$ & 108.37 & -132.91 \\
\hline $626.716^{a} 0.306$ & -85.00 & 141.40 & $1671.828^{c} 0.802$ & 153.10 & -200.44 \\
\hline $1299.883^{c} 0.430$ & -7.56 & -5.01 & $1672.865^{c} 0.987$ & -12.61 & -10.06 \\
\hline $1300.882^{c} 0.608$ & 94.15 & -107.22 & $1673.878^{c} 0.168$ & -168.93 & 211.74 \\
\hline $1301.883^{c} 0.787$ & 152.44 & -201.56 & $2037.812^{c} 0.110$ & -172.15 & 221.33 \\
\hline
\end{tabular}

studied system. The aim is to bring its orbital and physical parameters on firm ground. As mentioned above, accurate ephemeris and a detailed knowledge of the orbital and physical properties of a system are indeed crucial ingredients for interpreting the X-ray data and for uncovering the possible signature of a wind interaction.

The remaining of this paper is organised as follows. The next section describes the observational material and the data reduction processes. Sect. 3 aims at unveiling the discrepancies of the previously published orbital solutions. Sect. 4 discusses the physical properties and evolutionary status of HD 152218 and, in Sect. 5] we analyse the X-ray observations in the light of the upgraded orbital solution. Finally Sect. 6 provides a summary of the present work.

\section{Observations and data reduction}

\subsection{Optical spectroscopy}

The present work is based on 42 high-resolution spectra of HD 152218 obtained at the European Southern Observatory (ESO, La Silla, Chile) and at the Cerro Tololo Inter-American Observatory (CTIO). These were acquired during 35 different nights distributed over 8 runs between May 1997 and May 2004. The journal of the observations is presented in Table 1 .

In May 1997, five (resp. six) high resolution spectra of the He I 14471 (resp. He II 14686) line were obtained with ESO’s $1.4 \mathrm{~m}$ Coudé Auxiliary Telescope (CAT) at La Silla, using the Coudé Echelle Spectrometer (CES) equipped with the Long Camera (LC). The detector used was ESO CCD\#38, a Loral $2688 \times 512$ pixel CCD with a pixel size of $15 \times 15 \mu \mathrm{m}$. The slit width was chosen to achieve a nominal resolving power of 70000-80000. The effective resolving power as derived from the FWHM of the lines of the ThAr calibration exposures is 65000-75000. Typical exposure times were of the order of 30 minutes and the average signal-to-noise ratio (SNR) is about 140. The observed wavelength domain is centered on the He I 14471 or He II 14686 line and is $\sim 45 \AA$ wide.

Another set of three echelle spectra over the range 3850 to $5790 \AA$ was obtained with the Bench-Mounted Echelle Spectrograph (BME) attached to the $1.5 \mathrm{~m}$ CTIO Ritchey-Chrétien Telescope, during a 5-night run in June 1999. Forty nine orders were observed using the KPGL2 316 lines $\mathrm{mm}^{-1}$ grating as a cross-disperser. The detector was a Tek 2048 CCD with $24 \mu \mathrm{m}$ pixels. The slit width was set to $70 \mu \mathrm{m}$ corresponding to a resolving power of 45000 . Exposure times ranged from 45 to $60 \mathrm{~min}$, yielding a SNR in the continuum between 40 and 50 .

Between April 1999 and May 2002, we collected 20 echelle spectra covering the whole optical range $(\sim 3750$ $9200 \AA$ ) using the Fiber-fed Extended Range Optical Spectrograph (FEROS), an echelle spectrograph mounted at the ESO 1.5m telescope at La Silla. In May 2003 and May 2004, respectively three and five other FEROS spectra were obtained at the ESO $2.2 \mathrm{~m}$ telescope, at La Silla too. The detector was a $2 \mathrm{k} \times 4 \mathrm{k}$ EEV CCD with a pixel size of $15 \mu \mathrm{m} \times 15 \mu \mathrm{m}$. The spectral resolving power of FEROS is 48000 . Typical exposure times ranged, at the ESO $1.5 \mathrm{~m}$ telescope, from 10 to $20 \mathrm{~min}$ according to the weather conditions, resulting in typical SNR around 150. At the 


\begin{tabular}{rrr} 
& \multicolumn{3}{c}{ Table 1. Continue } \\
\cline { 2 - 3 } HJD $\phi_{\mathrm{HeI}} \overline{R V_{\lambda, 1}-\gamma_{\lambda, 1}} \overline{R V_{\lambda, 2}-\gamma_{\lambda, 2}}$ \\
\hline $2037.903^{c} 0.126$ & -174.37 & 222.99 \\
$2039.800^{c} 0.465$ & -3.87 & -1.32 \\
$2040.859^{c} 0.654$ & 111.51 & -142.01 \\
$2381.695^{c} 0.474$ & -2.42 & 0.13 \\
$2382.692^{c} 0.652$ & 109.41 & -147.06 \\
$2383.691^{c} 0.831$ & 149.50 & -193.52 \\
$2782.709^{d} 0.033$ & -98.31 & 129.27 \\
$2783.748^{d} 0.219$ & -148.99 & 194.69 \\
$2784.699^{d} 0.389$ & -17.66 & -15.11 \\
$3130.843^{d} 0.156$ & -173.97 & 223.41 \\
$3131.716^{d} 0.312$ & -85.25 & 133.87 \\
$3132.730^{d} 0.493$ & 4.66 & 3.89 \\
$3133.760^{d} 0.677$ & 115.83 & -163.34 \\
$3134.723^{d} 0.849$ & 141.10 & -189.24 \\
\hline ESO 1.5m; ${ }^{d} \mathrm{FEROS}^{-1}+\mathrm{ESO} / \mathrm{MPG} 2.2 \mathrm{~m}$ & $;{ }^{c} \mathrm{FEROS}$
\end{tabular}

ESO $2.2 \mathrm{~m}$ telescope, the SNR is about 200 in May 2003 and about 250 in May 2004. Typical exposure times at the ESO $2.2 \mathrm{~m}$ were of $13 \mathrm{~min}$.

The CES data were reduced in a standard way using the MIDAS package supported by ESO. The spectra were rectified by means of an instrumental response curve built from the observations, obtained under similar conditions, of a metal-poor 'reference' star (HD 203608: F6V). Finally, the spectra were normalized by fitting a low order polynomial to the continuum.

The BME data were reduced using the IRAF 1 package and following the recommendations of the BME User's Manual. The pixel to pixel variations were removed using flat field exposures taken with a very bright light source and a diffusing screen placed inside the spectrograph (so-called milky flats). A first rectification of the extracted echelle orders was carried out with the projector flat exposures. The spectra were then normalized by fitting a low-order polynomial to the continuum.

FEROS data were reduced using an improved version of the FEROS context (see details in Sana et al., 2003) working under the MIDAS environment. We mainly used the normalized individual orders. However, when doubts about the quality of the normalisation arise, we checked our results with the automatically merged spectrum.

\subsection{X-ray observations}

In September 2001, the NGC 6231 open cluster was the target of an X-ray observing campaign with the XMM-
Newton European observatory (Jansen et al., 2001). The campaign, of a total duration of about $180 \mathrm{ks}$, is described in length in Sana et al. (2006b) together with the data reduction and source identification. We only give a brief overview here, focusing on the additional elements specific to HD 152218.

The campaign actually consisted of six separate observations spread over 5 days. All three EPIC instruments (Strüder et al., 2001; Turner et al., 2001) were operated in the Full Frame mode together with the Thick Filter to reject UV/optical light. The field of view (FOV) of the EPIC instruments was centered on the colliding wind binary HD 152248, from which HD 152218 is separated by about $6 ! 8$. Due to the brightness of the cluster objects in the FOV, the Optical Monitor (Mason et al., 2001) was switched off throughout the campaign. Fig. 1 presents a view of the combined MOS images around HD 152218 while Table 2 provides the journal of the X-ray observations of the system.

HD 152218 unfortunately fell on a gap of the pn instrument and, as in Sana et al. (2006b), we only considered the EPIC MOS data. To obtain the averaged count-rates during the different pointings, we adopted the source positions given in the catalogue of Sana et al. (2006b) and we performed a psf (point spread function) fit using the SAS task emldetect. As in the previous works, we restrained our scientific analysis to the $0.5-10.0 \mathrm{keV}$ band and we adopted three energy sub-ranges: a soft $\left(\mathrm{S}_{\mathrm{X}}\right)[0.5: 1.0 \mathrm{keV}]$, a medium $\left(\mathrm{M}_{\mathrm{X}}\right)[1.0: 2.5 \mathrm{keV}]$ and a hard $\left(\mathrm{H}_{\mathrm{X}}\right)[2.5-10.0 \mathrm{keV}]$ band.

Located slightly offset from the cluster core, HD 152218 is reasonably well isolated in the X-ray images. To extract the source spectra, we used an extraction radius of $20^{\prime \prime} 2$, centered on the source position for all six pointings and for the two EPIC MOS instruments. The different backgrounds were estimated from source-free regions located on the same CCD detector as HD 152218 (CCD \#3 and 5 respectively for the MOS1 and MOS2 instruments). The adopted source extraction region is shown in Fig. 1. Displays of the corresponding background extraction regions are presented in Sana et al. (2006c) together with their positions and sizes.

Using these regions, we extracted the source spectra from the two MOS instruments and for the six pointings individually. We also extracted the merged spectra in each instrument, thus combining the six observations of HD 152218. For this purpose, we built the corresponding Ancillary Response Files (the so-called arf files) using the SAS task arfgen. We adopted the Redistribution Matrix Files ( $r m f$ ) provided by the SOC. The spectra were finally binned to reach at least 10 counts per bin. The X-ray data will be discussed in Sect. 5 .

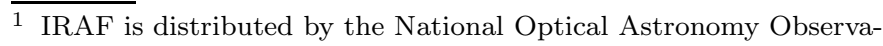
tories.
} 
Table 2

Journal of the XMM-Newton observations of HD 152218. The Julian Date (JD) at mid-exposure is given in Col. 2 . Cols. 3 and 4 list the effective exposure times for the EPIC MOS1 and MOS2 instruments while Cols. 5 and 6 report the background-subtracted, vignetting and exposure-corrected count-rates in the different instruments. The last column provides the orbital phase of HD 152218 for each XMM-Newton observation at mid-exposure, according to the HeI-line ephemerides given in Table 4 The phase extension of the pointings is about 0.06 . Obs. 2 and 4 were however affected by high background events that reduced the nominal exposure time by about one third.

\begin{tabular}{ccccccc} 
Obs. \# & \multicolumn{5}{c}{ JD } & \multicolumn{5}{c}{ Effective duration $(\mathrm{ks})$ Count rates $\left(10^{-3} \mathrm{cnt} \mathrm{s}^{-1}\right)$ Phase $\phi_{\mathrm{HeI}}$ of } \\
& JD-2 450 000 MOS1 & MOS2 & MOS1 & MOS2 & HD 152218 \\
\hline 1 & 2158.214 & 33.1 & 33.2 & $13.3 \pm 0.8$ & $14.7 \pm 0.9$ & 0.591 \\
2 & 2158.931 & 19.8 & 19.8 & $17.5 \pm 1.2$ & $12.9 \pm 1.0$ & 0.719 \\
3 & 2159.796 & 33.7 & 33.9 & $13.8 \pm 0.8$ & $13.1 \pm 0.8$ & 0.873 \\
4 & 2160.925 & 26.0 & 24.3 & $12.5 \pm 0.9$ & $11.6 \pm 0.9$ & 0.075 \\
5 & 2161.774 & 30.9 & 31.0 & $14.4 \pm 0.9$ & $13.4 \pm 0.9$ & 0.226 \\
6 & 2162.726 & 32.9 & 32.8 & $15.7 \pm 0.9$ & $14.4 \pm 0.9$ & 0.396 \\
\hline
\end{tabular}

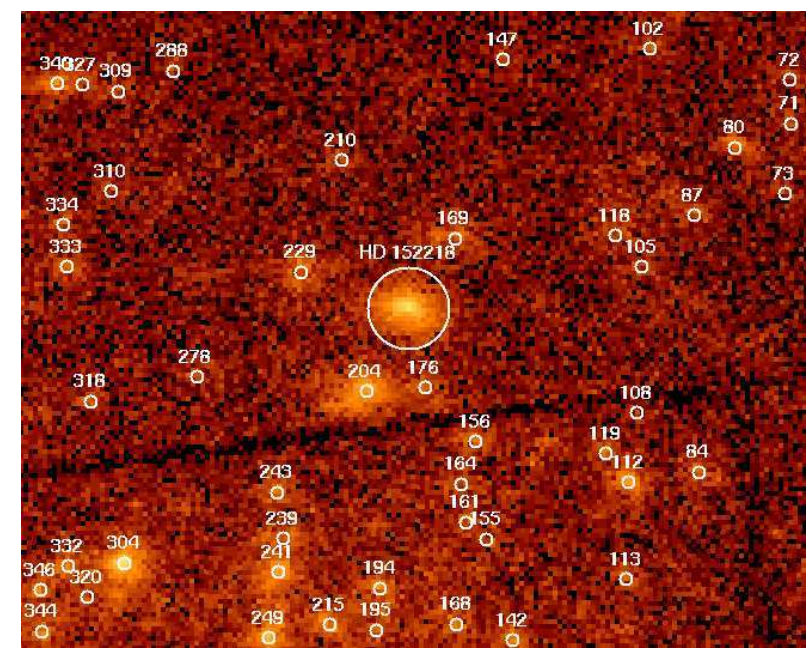

Fig. 1. Combined EPIC MOS1 + MOS2 image in the vicinity of HD 152218. The adopted source extraction region is shown. Neighbouring $\mathrm{X}$-ray sources are labelled using the internal numbering of Sana et al. (2006b).

\section{Unveiling the correct orbital solution}

\subsection{Optical spectrum}

The spectrum of HD 152218 is clearly dominated by the usual Balmer, He I and He II lines seen in absorption (Fig. 2). Beside these, the spectrum reveals metallic lines of N III, Si III, Si IV, C IV and O III that are typical of O-type stars. Only the C III 15696 line is seen in emission. Most of the observed stellar lines are double (see e.g. Fig. 3) and display clear Doppler shifts. The HD 152218 spectrum also reveals a number of diffuse interstellar bands (DIBs), as well as interstellar absorptions due to $\mathrm{NaI}, \mathrm{Ca}$, $\mathrm{Ca}$ II, $\mathrm{CH}$ and $\mathrm{CH}^{+}$.

We measured the Doppler shifts by fitting a sum of Gaussians to the different line profiles. Whenever the components get closer to conjunction, the disentangling of their spectral signature was more difficult. To get reliable results we had to constrain the full width at half maximum
(FWHM) of the fitted Gaussian profiles. We thus computed the mean FWHMs of the primary and secondary lines according to the measurements performed near quadrature. Considering the properties of the studied lines, we then decided either to fix the line FWHMs to their mean value or to request the primary and secondary lines to have equal FWHMs. The resulting radial velocities (RVs) were assigned a lower weight in the determination of the orbital solution (see Sect. 3.3). In order to get a reliable solution, we considered 11 different lines: He I łł4026, 4388, 4471, 4922, 5016, 5876, 7065, He II ł 4542, 4686, 5412 and O III 15592. Note that the He II $\lambda \lambda 4542$ and 5412 lines were rarely deblended and show a faint secondary component only. For these lines, we thus focused on the primary orbital motion. We also note that the O III 15592 line is only seen in the primary spectrum. Finally, to compute the RVs associated with the measured Doppler shifts, we adopted the effective wavelengths for O-stars from Conti et al. (1977) below $4800 \AA$ and from Underhill (1994) above.

\subsection{Period determination}

Our period search relies on the method of Lafler \& Kinman (1965, L\&K) and on the Fourier-analysis technique of Heck et al. (1985, HMM85, see also Gosset et al. 2001 for comments) applied on the different data sets of Table 3. Using the L\&K algorithm, the obtained periods are in excellent agreement, yielding a mean value of $5.60380 \pm 0.00005 \mathrm{~d}$. The results from the Fourier analysis are more conflicting. The He ı 4471 and He II $\nmid 4686$ data sets yield a period of 5.604 days while the other lines indicate 5.519 days. An inspection of the corresponding periodograms revealed that the period determination is strongly affected by a 1-year aliasing, leading to a possible confusion between orbital frequencies of 0.1784 and $0.1812 \mathrm{~d}^{-1}$. These latter values correspond respectively to the two period values quoted here above. From Fig.4(left), it is clear that the period can not be reliably constrained from the sole FEROS data. The inclusion of the CES and, to a lesser extent, of the BME data clearly improves the 

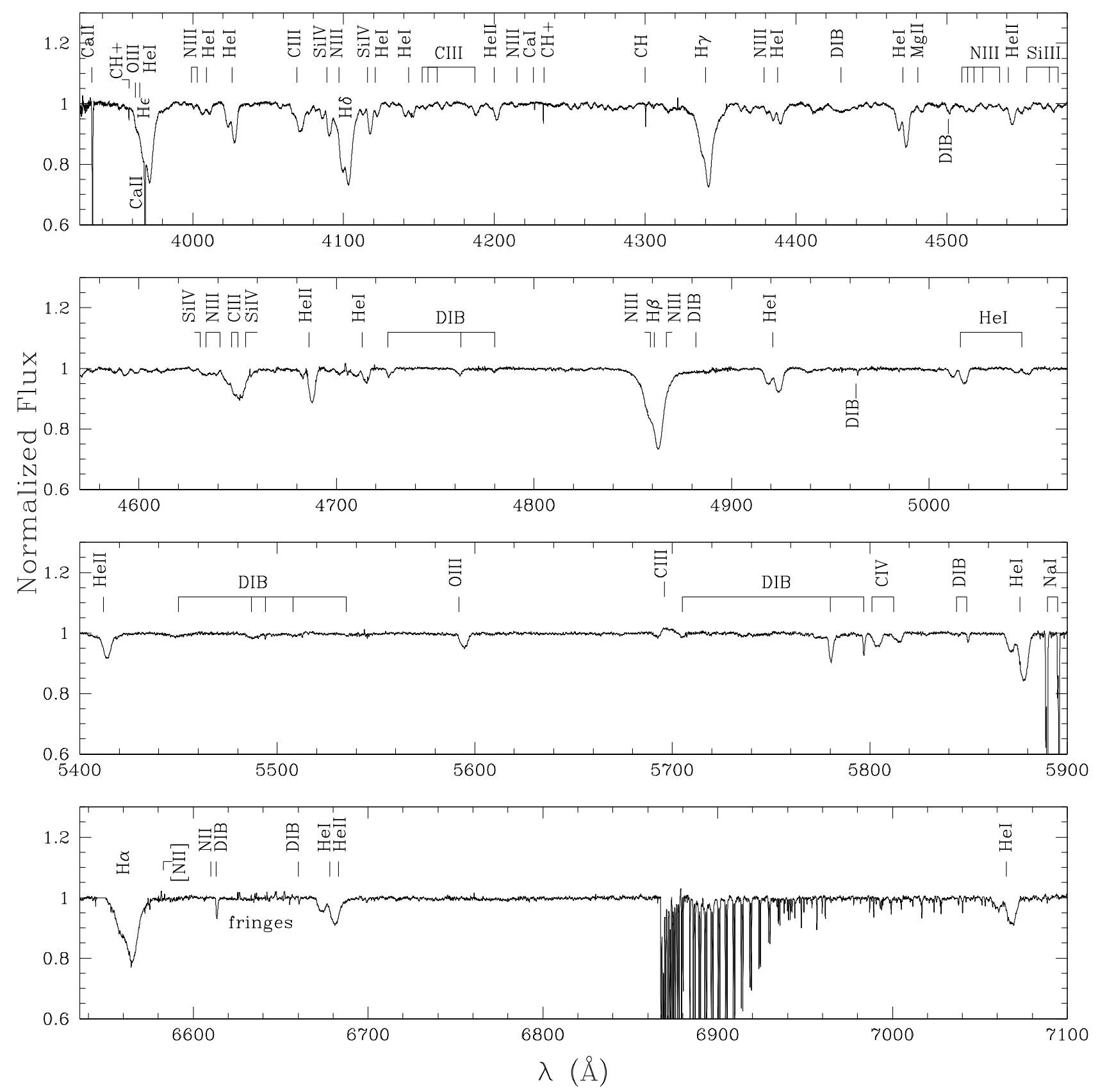

Fig. 2. Spectrum of HD 152218 as observed with the FEROS spectrograph on HJD 2453134.723 ( $\phi \sim 0.85)$. At this orbital phase, the spectrum of the most massive star is shifted towards the red. The most prominent lines are indicated at their rest wavelengths. 


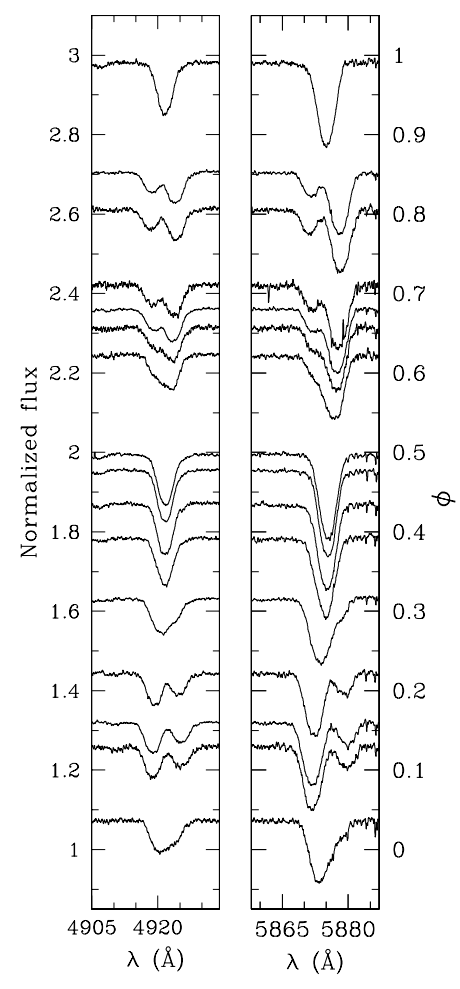

Fig. 3. He I 14922 (left) and He I 15875 (right) lines at selected phases. The spectra were shifted along the vertical axis according to the value of their phase (right-hand scale). The secondary spectral signature is clearly identified on most spectra but the blended ones.
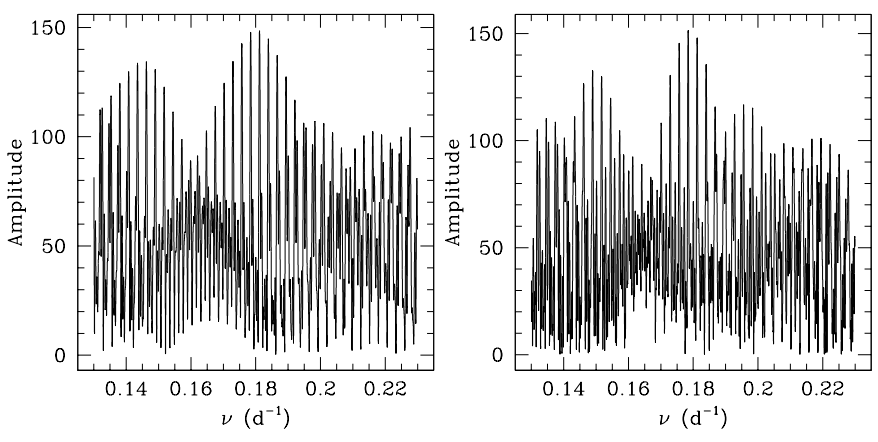

Fig. 4. Left panel: HMM85 periodogram (i.e. amplitude spectrum) associated with the FEROS He I 14471 primary data set. Periodograms associated with other lines are very similar. Right panel: idem, using the CES and BME measurements in addition to the FEROS data. This latter data set favors a period of $5.604 \mathrm{~d}$, corresponding to $\nu \approx 0.1784 \mathrm{~d}^{-1}$.

situation (Fig. 4. right) but still does not allow to draw a firm conclusion.

Our data sets consist of spectra spread over 2500 days for the He I 44471 and He II $\$ 4686$ lines or over 1800 days for the other lines. These observational time bases correspond respectively to natural peak widths $\Delta \nu$ of $0.0004 \mathrm{~d}^{-1}$ and $0.0006 \mathrm{~d}^{-1}$. The full width at half maximum of the actual peaks in the periodogram is in agreement with these theoretical values. Adopting an uncertainty of one tenth of the peak width, we respectively obtained $\sigma_{P}=0.0013$ or $0.0017 \mathrm{~d}$. These values however only account for the uncertainty due to the finite width of the peak, but do not
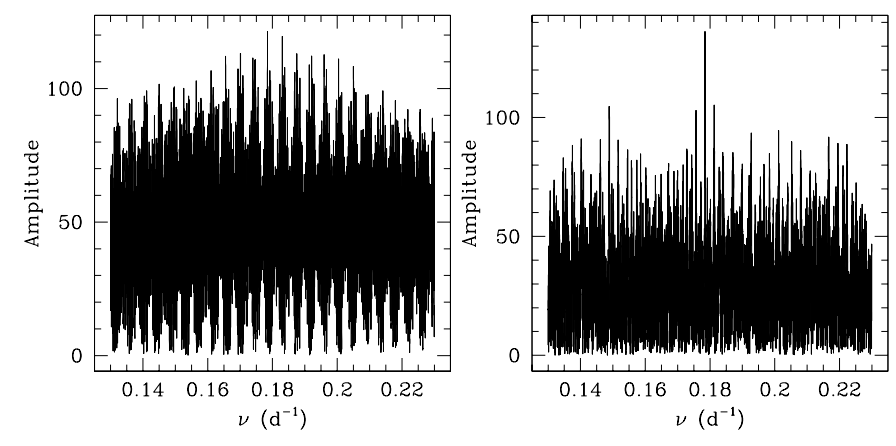

Fig. 5. Left panel: HMM85 periodogram corresponding to the IUE+optical data sets used by SLP97. Right panels: idem, combining our data with published data sets.

consider the possible confusion between different aliases.

To solve the aliasing problem, we turned to the literature. As Hill et al. (1974, HCB74) and Levato \& Morrell (1983, LM83) derived quite different period values compared to the ones quoted above, we first applied our period search methods using their data sets and found a very large number of aliases covering a frequency range corresponding to period values between about 5 and 6.7 days. Their proposed period values were thus much more poorly constrained than what their error-bars suggested. On the other hand, Stickland et al. (1997, SLP97) obtained a pe$\operatorname{riod} P \approx 5.604 \mathrm{~d}$, very similar to one of our aliases. We used both the L\&K and HMM85 algorithms on the different data sets considered by SLP97 and we re-derived very similar values, except for the related uncertainties. Adopting a typical error corresponding to one tenth of the periodogram peak width, we obtained values about 6 times larger. The periodogram (Fig. 5. left panel) also indicates a possible confusion with a neighbouring alias at $\nu \approx 0.183 \mathrm{~d}^{-1}$. The period value is again not so clearly established. We however note that this latter alias is not present in our data set. By combining the different data sets, we could thus hope to definitely constrain the period.

To combine our data with other observational data sets, we first averaged the RV measurements obtained from the different lines quoted in Table ${ }^{2}$. Then, applying the L\&K and HMM85 period searches to all the available RV measurements, we derived values of $P=5.6039$ and $5.6040 \mathrm{~d}$ respectively. The corresponding Fourier periodogram is displayed in Fig. 5 (right) and clearly shows that the orbital peak is now well isolated. The time span of the whole observational set is $\sim 21915 \mathrm{~d}$, yielding a theoretical periodogram peak width of $\Delta \nu=4.6 \times 10^{-5} \mathrm{~d}^{-1}$. The observed width of the peak agrees with this value and yields $\Delta P=$ $1.4 \times 10^{-3} \mathrm{~d}$. We adopted a final uncertainty corresponding to one tenth of the peak width, i.e. $\sigma_{P}=1.4 \times 10^{-4}$ d.

\footnotetext{
2 In this first approach, we did not correct the individual line RVs for their systemic velocities but we rather performed a direct average. Indeed the systemic velocities should be obtained from RV curve fitting. They thus require an first accurate estimate of the period
} 


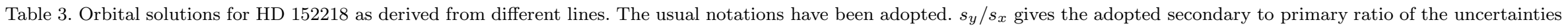
on the measured RVs (see Sect. 3.3). The quoted error-bars are the 1- $\sigma$ uncertainties.

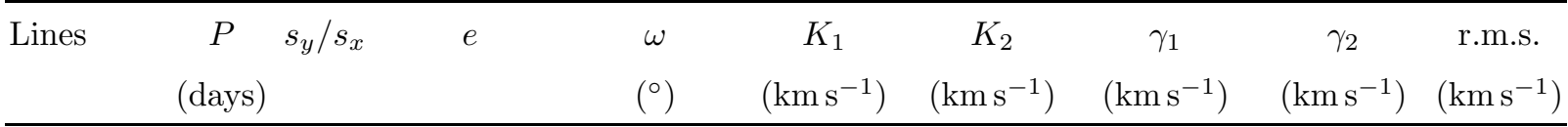

He Ił4026 $5.604001 .4 \quad 0.268 \pm 0.007104 .4 \pm 1.7164 .4 \pm 1.3209 .2 \pm 1.6-16.7 \pm 1.4-17.3 \pm 1.6 \quad 5.6$

He Ił4388 $5.60395 \quad 1.2 \quad 0.253 \pm 0.011 \quad 99.4 \pm 3.3159 .2 \pm 2.2208 .1 \pm 2.8-11.4 \pm 2.3-15.5 \pm 2.7 \quad 9.5$

He Ił4471 $5.60386 \quad 1.7 \quad 0.242 \pm 0.008101 .7 \pm 2.6160 .2 \pm 1.6215 .0 \pm 2.2-26.4 \pm 1.7-30.6 \pm 2.1 \quad 7.7$

He II $¥ 46865.60391 \quad 1.7 \quad 0.275 \pm 0.008108 .4 \pm 1.8165 .8 \pm 1.7207 .9 \pm 2.2-15.4 \pm 1.7-16.8 \pm 1.9 \quad 7.1$

He Ił4922 $5.60393 \quad 1.4 \quad 0.261 \pm 0.008103 .0 \pm 2.1159 .8 \pm 1.4214 .4 \pm 1.8-18.0 \pm 1.6-21.8 \pm 1.9 \quad 6.3$

He I $150165.60405 \quad 1.6 \quad 0.269 \pm 0.010102 .6 \pm 2.4167 .4 \pm 2.0215 .2 \pm 2.6-16.3 \pm 2.0-17.2 \pm 2.4 \quad 8.3$

He I $158765.60410 \quad 1.6 \quad 0.271 \pm 0.006104 .5 \pm 1.5162 .4 \pm 1.1221 .4 \pm 1.5-21.9 \pm 1.2-21.1 \pm 1.5 \quad 4.8$

He I $170655.60410 \quad 1.7 \quad 0.255 \pm 0.008101 .0 \pm 2.2165 .1 \pm 1.6214 .0 \pm 2.1-17.1 \pm 1.7-11.7 \pm 2.0 \quad 6.4$

He IIł4542 $5.60400-0.288 \pm 0.014101 .3 \pm 2.2160 .9 \pm 2.1 \quad-\quad-21.1 \pm 1.2 \quad-\quad-2 \quad 6.5$

He II $154125.60419-0.302 \pm 0.011104 .0 \pm 1.6161 .4 \pm 1.7 \quad-\quad-16.5 \pm 1.0 \quad-\quad-\quad 0.2$

$\begin{array}{lllllll}\text { O III } 55925.60409 & - & 0.274 \pm 0.012106 .1 \pm 2.0162 .7 \pm 1.9 & - & -17.1 \pm 1.7 & - & 6.0\end{array}$ 


\subsection{Orbital elements}

Now that the period is reliably constrained, we can derive the orbital parameters of the system by fitting the observed RV-curves assuming a Keplerian motion for the two components. We first computed a series of orbital solutions using the RV sets associated with the different absorption lines listed in Table 3. For SB1 lines, we used the algorithm of Wolfe et al. (1967) in its original form and assigned the same weight to each measurement. For the data sets associated with SB2 lines, we used a modified version of the algorithm, adapted to SB2 systems as explained in Sana et al. (2006a), and we only considered those data points for which the two components could be measured with a reasonable accuracy. We assigned a half weight to those RV points for which the FWHMs had to be constrained (see Sect. 3.1). Because of the lower SNR of the BME data, we gave them a weight of one fourth while computing the orbital solution. In the observed wavelength domain, the BME measurements were further not included for the fainter lines nor for the lines more difficult to disentangle (He I $\lambda \lambda 4388$, 5016, He II 14542, O III 15592). In our derived solutions, we adopted a period value and a relative primary to secondary weight ratio $\left(s_{y} / s_{x}\right)$ that yield the lowest $\chi^{2}$. These best solutions, including a reappraisal of the period 3 , are presented in Table 3 together with the corresponding rootmean-square (r.m.s) residuals of the fit.

In a next step, we computed the mean RVs of the SB2 He I-lines quoted in Table 3 as well as of the sole primary lines. For this purpose, we first shifted the individual RVs to a common frame, by subtracting the individual systemic velocities. The resulting mean RVs are listed in Table 1. The orbital solutions computed using the averaged primary (SB1) and the averaged He I-line (SB2) RV measurements are given in Table 4 together with the physical parameters of the system. Fig. [6 displays the RV curves corresponding to the averaged SB2 solution. The latter solution will be adopted throughout the rest of the paper to constrain the physical properties of the HD 152218 components (see Sect. 4). Corresponding primary and secondary apparent systemic velocities, as obtained from a weighted mean of the values quoted in Table 3, are $\overline{\gamma_{1}}=-18.7 \pm 0.6 \mathrm{~km} \mathrm{~s}^{-1}$ and $\overline{\gamma_{2}}=-19.2 \pm 0.7 \mathrm{~km} \mathrm{~s}^{-1}$.

Our final SB2 solution is significantly different from the most recent determination by García \& Mermilliod (2001, GM01) who obtained $P \sim 4.9 \mathrm{~d}$ and $e \sim 0.4$. We however mostly recover, within the error-bars, the results of an older study by SLP97 Indeed, our new values fall within 3$\sigma$ from the results of SLP97, though with a slightly smaller eccentricity and larger RV-curve semi-amplitudes. Only the longitude of the periastron seems to differ significantly: $80.6 \pm 2.1$ for SLP97 versus $104.2 \pm 1.6$ for our solution. This suggests the presence of an apsidal motion of the or-

\footnotetext{
3 The period has been adjusted simultaneously together with the other orbital parameters, adopting the values derived in Sect. 3.2 as initial guess.
}

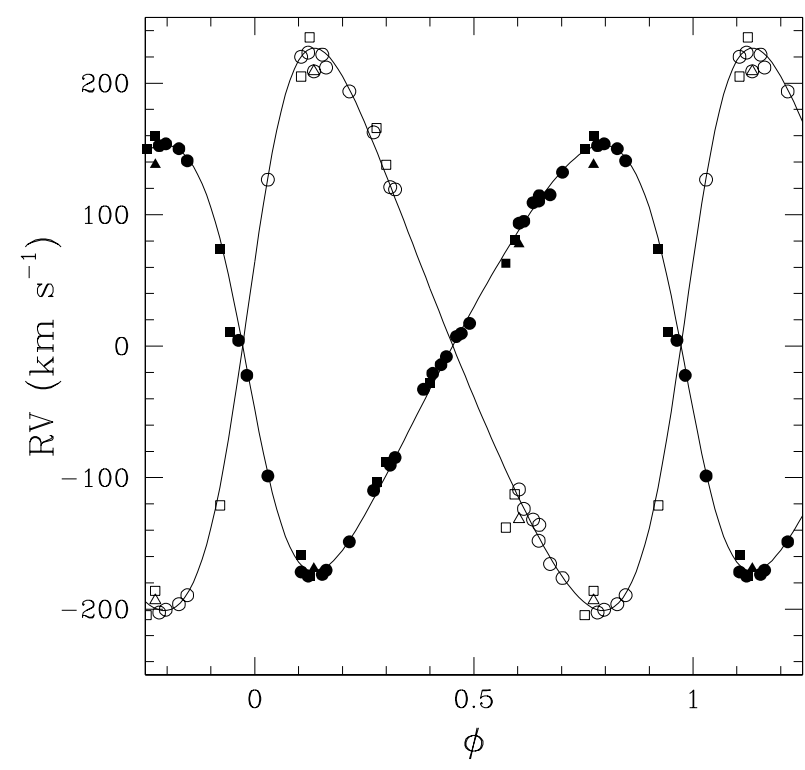

Fig. 6. HD 152218 RV-curves corresponding to the HeI solution of Table 4 Note that the respective systemic velocities have been subtracted. RV measurements listed in Table 1 have been overplotted. Filled and open symbols are for the primary and secondary components respectively. Different symbols indicate different instruments used: CES, squares; BME, triangles; FEROS, circles.

Table 4

Orbital and physical parameters of HD 152218 as deduced from two different data sets: the averaged RVs computed over selected primary SB1 lines (Col. 2) and over He I SB2 lines (Col. 3). The usual notations have been adopted. $T_{0}$ is the time of periastron passage and is adopted as phase zero (i.e. $\phi=0.0$ ). Note that the primary and the HeI lines solution were computed in the systemic velocity frame.

\begin{tabular}{ccc}
\hline & Prim. & He I lines \\
\hline$P(\mathrm{~d})$ & 5.60404 & 5.60391 \\
$s_{y} / s_{x}$ & $\mathrm{n}$. & 1.5 \\
$m_{1} / m_{2}$ & $\mathrm{n}$. & $1.319 \pm 0.014$ \\
$e$ & $0.275 \pm 0.008$ & $0.259 \pm 0.006$ \\
$\omega\left({ }^{\circ}\right)$ & $104.2 \pm 1.6$ & $104.0 \pm 1.6$ \\
$T_{0}(\mathrm{HJD}$ & 3196.493 & 3197.229 \\
$-2450000)$ & \pm 0.024 & \pm 0.025 \\
$K_{1}\left(\mathrm{~km} \mathrm{~s}^{-1}\right)$ & $162.4 \pm 1.1$ & $162.2 \pm 1.2$ \\
$K_{2}\left(\mathrm{~km} \mathrm{~s}^{-1}\right)$ & $\mathrm{n}$. & $213.9 \pm 1.5$ \\
$\gamma_{1}\left(\mathrm{~km} \mathrm{~s}^{-1}\right)$ & $0.7 \pm 0.9$ & $0.9 \pm 1.2$ \\
$\gamma_{2}\left(\mathrm{~km} \mathrm{~s}^{-1}\right)$ & $\mathrm{n}$. & $-0.6 \pm 1.4$ \\
$a_{1} \sin \left(\mathrm{R}_{\odot}\right)$ & $17.28 \pm 0.13$ & $17.34 \pm 0.13$ \\
$a_{2} \sin i\left(\mathrm{R}_{\odot}\right)$ & $\mathrm{n}$. & $22.87 \pm 0.17$ \\
$m_{1} \sin ^{3} i\left(\mathrm{M}_{\odot}\right)$ & $\mathrm{n}$. & $15.82 \pm 0.26$ \\
$m_{2} \sin ^{3} i\left(\mathrm{M}_{\odot}\right)$ & $\mathrm{n}$. & $12.00 \pm 0.19$ \\
r.m.s. $\left(\mathrm{km} \mathrm{s}^{-1}\right)$ & 4.1 & 6.1 \\
\hline
\end{tabular}

der of $20^{\circ}$ over about $7 \mathrm{yr}$. This question is addressed in the next section.

\subsection{Apsidal motion}

As already mentioned, several authors have collected a number of spectroscopic observations of HD 152218 and measured the RVs of its two components. The largest sets are due to Struve (1944), HCB74, SLP97 and GM01 who respectively obtained 5, 15, 22 and 7 spectra. Struve pub- 


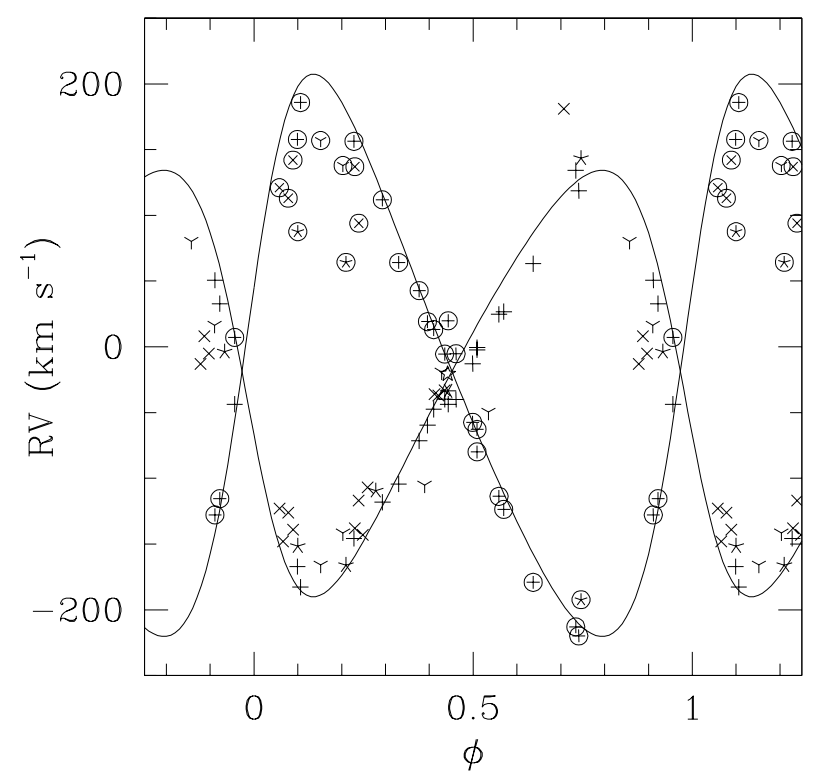

Fig. 7. Literature RV measurements plotted over the HeI SB2 RV-curves of Fig. [6 shifted according to their respective apparent systemic velocities. The phases were computed using the He I ephemeris of Table 4 Different symbols indicate different works: (five branch star) Struve (1944); $(\times)$ HCB74; (asterisk) LM83; (open five-branch star) Conti et al. (1977); (+) SLP97; (Y) corrected GM01. These symbols inscribed in open circles indicate secondary RV measurements.

lished the observing dates using universal times with day and month. HCB74 later presented corresponding Julian dates (JD). We however note that, though quoted otherwise, these dates are not heliocentric Julian dates (HJD) but simply JD. We thus recomputed the appropriate HJD before using the Struve data set. A word of caution is also required for the GM01 observations. As already mentioned above, GM01 quoted erroneous HJDs for part of their observation 4 . We thus corrected the corresponding values prior to the inclusion of the GM01 observations. We completed these four sets by an additional measurement from Conti et al. (1977) and another one by LM83. We finally added the RVs from the present work (obtained as the average of the $\gamma$-corrected individual lines measurements) and we adopted the systemic velocities derived in Sect. 3.3 .

In a first approach, Fig. 7 shows the literature measurements plotted over the He I orbital solution of Table4. The secondary measurements present a larger scatter and we will thus focus on the primary data for the rest of this section. In Fig. 7 we also observe systematic shifts between the literature measurements and our newly derived orbital solution. As suggested in the previous section, this most probably comes from the presence of an apsidal motion. Fixing the period and the eccentricity at the values quoted in Table 4 for the He I-solution, we adjusted the different data sets of Table 5 and derived, for each epoch,

\footnotetext{
${ }_{4}$ In [GM01]'s Table 2, the HJDs around 2449910 are probably correct, those around 2450590 are overestimated by exactly one day for observations obtained after midnight UT. We could not check the more recent observations obtained by GM01 at HJDs 2 451360 .
}

Table 5

Best fit values for the longitude of the periastron $\omega$ at different epochs. The data sets labelled ' $\mathrm{A}$ ' to ' $\mathrm{C}$ ' are described in the text.

\begin{tabular}{|c|c|c|c|}
\hline \multicolumn{4}{|c|}{ Data set $\overline{\mathrm{HJD}-2400000} \omega\left(^{\circ}\right)$ r.m.s. $\left(\mathrm{km} \mathrm{s}^{-1}\right)$} \\
\hline Struve (1944) & 31223.5 & -100 & 5.4 \\
\hline $\mathrm{HCB} 74$ & 40818.9 & 34 & 11.7 \\
\hline SLP97 $^{\mathrm{a}}$ & 49627.5 & 82 & 7.6 \\
\hline This work & 51652.1 & 102 & 6.6 \\
\hline A & 49333.8 & 72 & 19.9 \\
\hline B & 50861.8 & 95 & 11.8 \\
\hline $\mathrm{C}$ & 52621.3 & 106 & 4.2 \\
\hline
\end{tabular}

a. The first point of the $\overline{\text { SLP97 }}$ set is separated by about 3300 days from the other points of the set and was not included in the fit.

the best-fit longitude of periastron. These values are plotted in Fig. 8. We note that, using the RV sets of HCB74 and Stickland et al. (1997), we re-derived the longitude values respectively quoted in their proposed orbital solutions. We also built three subsets of data using the data of GM01, SLP97 and ours. These sets, labelled A, B and $\mathrm{C}$ in Table 5, correspond respectively to data obtained before HJD $=2450$ 000, in-between HJD = 2450000 and HJD $=2451800$ and after HJD = 2452 000. These sets are however not independent from the sets labelled 'SLP97' and 'This work' in Table 5 as they are formed by the same observations. We consider our approach to provide a kind of moving average over the time. It can also be seen as a way to give more weight to the more recent data, that probably provide a better accuracy than the older sets of Struve and HCB74. Using a linear regression, we then computed the best-fit linear relation that reproduces the observed values, the slope of which provides the rate of the apsidal motion. We obtained: $\dot{\omega}=9.1 \pm 0.8 \times 10^{-3} \mathrm{~d}^{-1}$, corresponding to about $\dot{\omega}=3.3 \pm 0.3 \mathrm{yr}^{-1}$. It is worth to note here, that, because of the similar $K_{1}$ and $K_{2}$, the shape of the RVcurve is little altered by a change of $\omega$ of $\pi$. By chance, thus, neither the Struve (1944) data nor those of HCB74 could strongly bias the orbital period determination of Sect. 3.2 . Indeed similar results are obtained while including or rejecting these two sets from the period search analysis.

A word of caution is however necessary concerning the rate of the apsidal motion. Indeed, we have also performed a similar analysis using our data only. These were grouped into five different subsets according to the different observing epochs. The apsidal motion is again clearly detected, even considering the comparatively smaller time span of our spectroscopic campaign. The obtained rate is however somewhat slower: $\dot{\omega}=1.4 \pm 0.6 \mathrm{yr}^{-1}$. Accounting in addition for the SLP97 data, we rather obtain $\dot{\omega}=3.0 \pm$ $0.6 \mathrm{yr}^{-1}$. Although there is little doubt left about the existence of an apsidal motion, the exact rate at which the latter occurs might still be uncertain. This probably reflect the difficulty to combine inhomogeneous data sets. 


\section{Physical parameters}

\subsection{Spectral types and luminosity classes}

The dominant spectral signature of the primary component in the spectrum of HD 152218 is characteristic of a late O-type star. We adopted the classification criteria from Conti (1973b) as adapted to late O-stars by Mathys (1988). They are based on the equivalent width (EW) ratio of the He I 14471 and He II 14542 lines. Doing this, we only considered the EWs measured on the spectra where the two components are disentangled. For the primary, we obtained a mean $\log W_{1}^{\prime}=\log W_{\lambda 4471}-\log W_{\lambda 4542}=0.33 \pm 0.06$ which corresponds to a spectral type O9, with spectral type O8.5 within $1 \sigma$. For the secondary, we obtained $\log W_{2}^{\prime}=$ $0.67 \pm 0.08$. This indicates an 09.7 star, with the O9.5 type at $1-\sigma$.

To determine the luminosity classes, we adopted the criterion from Conti \& Alschuler (1971) based on the EW ratio of the SiIV 14089 and He I 14144 lines. We respectively obtained $\log W_{1}^{\prime \prime}=\log W_{\lambda 4089}-\log W_{\lambda 4144}=$ $0.25 \pm 0.05$ and $\log W_{2}^{\prime \prime}=-0.12 \pm 0.13$. These values lead to giant and main sequence luminosity classes for the primary and secondary components respectively. According to Mathys (1988), we also measured $\log W_{1}^{\prime \prime \prime}=$ $\log W_{\lambda 4388}+\log W_{\lambda 4686}=5.03 \pm 0.04$. This corresponds to a supergiant if the primary brightness ratio $l_{1}=\frac{L_{1}}{L_{\text {tot }}}>0.76$, to a giant if $0.76>l_{1}>0.43$ and to a main sequence star otherwise. For the secondary, we get $\log W_{2}^{\prime \prime \prime}=4.22 \pm 0.15$. The respective critical values for $l_{2}=\frac{L_{2}}{L_{\text {tot }}}$ are this time 0.12 and 0.07 . Such a low contribution of the secondary to the total flux is improbable. Mathys criterion thus indicates a supergiant secondary, which is clearly at odds with the result of the Conti \& Alschuler (1971) criterion. However, the Mathys criterion can be biased if the He IIł4686 line is partly filled by emission produced e.g. in a wind interaction region, which is possibly the case in HD 152218 (see Sect. 5) The comparison of our spectra with the atlas of standards (Walborn \& Fitzpatrick, 1990) seems to favor a giant class for the primary star, but it is very difficult to reject the main sequence option. It also indicates that the secondary is most probably a main sequence star.

\subsection{Optical brightness ratio and evolutionary status}

We roughly estimated the optical brightness ratio based on the dilution of the primary and secondary lines in the spectrum of HD 152218. For this purpose, we compared the mean EWs of the primary and secondary lines with typical (averaged) EWs of respectively O9 (Conti \& Alschuler, 1971; Conti, 1973a; Mathys, 1988, 1989) and O9.7 stars (Mathys, 1988, 1989). We first compared the primary line strength with typical O9III EWs. Based on the He I łł4026, 4144, 4388, 4471, He II łł4542, 4686 and Si IV $¥ 4088$ lines, we obtained an averaged brightness ratio of $l_{1}=0.76 \pm 0.05$.

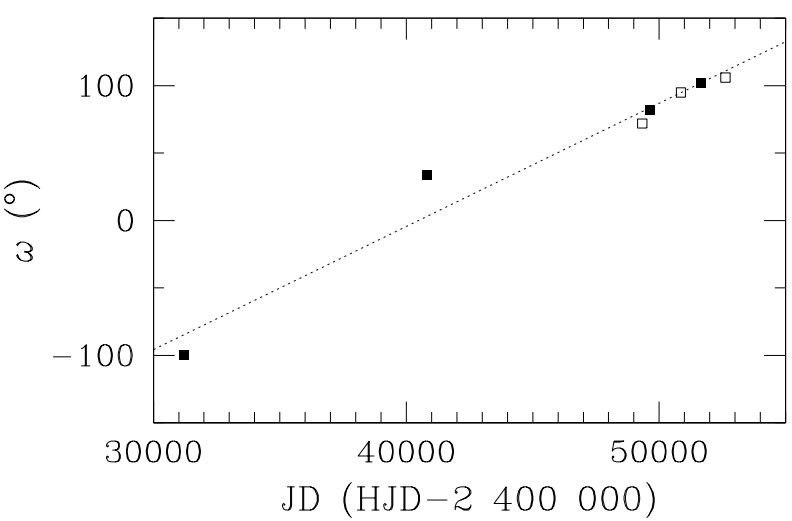

Fig. 8. Values of the argument of periastron $\omega$ computed for different epochs. The dotted line gives the best-fit relation, corresponding to an apsidal motion of $9.1 \pm 0.8 \times 10^{-3} \mathrm{~d}^{-1}$, or about $3.3 \pm 0.3 \mathrm{yr}^{-1}$.

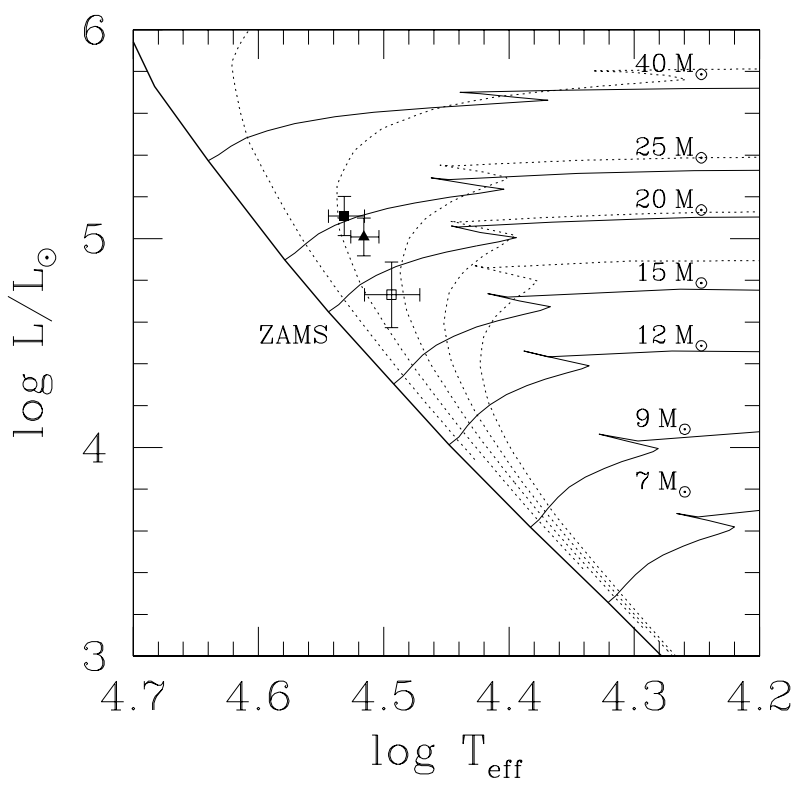

Fig. 9. HD 152218 primary (filled symbols) and secondary (open symbol) location in the H-R diagram. Triangle (resp. square) symbols indicate an adopted giant (resp. main sequence) luminosity class. The evolutionary tracks from Schaller et al. (1992) have been overplotted (plain lines) together with isochrones (dotted lines) computed for ages ranging from 2 to $10 \mathrm{Myr}$ with a step of $2 \mathrm{Myr}$.

Using typical O9V EWs yields a lower value $l_{1}=0.68 \pm 0.11$. We however note that the Si IV 14088 line is particularly strong in the primary spectrum and is the main cause for the large scatter obtained. Rejecting the Si IV 14088 line from the comparison with O9V typical EWs, we obtained $l_{1}=0.63 \pm 0.04$.

Turning to the secondary component, we also compared the secondary line strengths to the typical EWs reported by Mathys (1988, 1989), who only considered the He I $\lambda \lambda 4388,4471$ and He II $\lambda \lambda 4542,4686$ lines. We note that the He II $\$ 4686$ line provides results that clearly differ from the three other lines, but this is not surprising since the HeII 14686 line is particularly faint in the secondary spectrum. Mean values for the three other lines indicate $l_{2}=0.34 \pm 0.02$ and $0.41 \pm 0.05$ for dwarf and giant star respectively. The obtained values for the primary and sec- 
ondary contribution to the observed fluxes are thus in acceptable agreement with each other. They also agree with the results of Penny et al. (1994) who quoted a primary to secondary UV flux ratio of 2.0. In the following, we will adopt a primary to secondary brightness ratio corresponding to $l_{1}=0.67 \pm 0.10$, which reasonably well reproduces the results of the above discussion.

\subsection{Effective temperatures and radii}

Sung (2005, private communication) reported $V=7.562$ and $B-V=0.191$ for the system. Using $R=3.3$ (Sung et al., 1998), adopting the absolute colors quoted by Schmidt-Kaler (1982) and a distance modulus $D M=$ $11.07 \pm 0.04$ (Sana et al., 2006b, and references therein), preliminary computations indicate that the system is too faint to harbour a giant primary, unless its contribution $l_{1}$ to the observed flux brightness were very close to unity. This is clearly not confirmed by the previous discussion and we suggest that the primary in HD 152218 could be a class IV object. Correcting from the reddening and the distance effects, we obtained $M_{\mathrm{V}, 1}=-4.72 \pm 0.22$ and $M_{\mathrm{V}, 2}=$ $-3.95 \pm 0.37$. Because the intrinsic colour $(B-V)_{0}$ of an O-type star does almost not depend on the stellar gravity, we note that these values are free from the hypotheses on the luminosity class. According to Humphreys \& McElroy (1984), these values correspond to the primary being a V-IV star and the secondary, a V star. Adopting the Humphreys \& McElroy temperature scale and bolometric corrections, we derived $M_{\mathrm{bol}, 1}=-8.0 \pm 0.2$ (resp. $M_{\mathrm{bol}, 1}=$ $-7.8 \pm 0.2$ ) if the primary is a dwarf (resp. giant) star. We obtained $M_{\mathrm{bol}, 2}=-7.1 \pm 0.4$ for the main sequence secondary. Finally, using temperatures of $T_{\text {eff }}^{\text {O9III }}=32.8_{31.9}^{33.6} \mathrm{kK}$ (resp. $T_{\text {eff }}^{\mathrm{O} 9 \mathrm{~V}}=34.032 .0 \mathrm{kK}$ ), we derived a primary radius of $10.3 \pm 1.3 \mathrm{R}_{\odot}\left(\right.$ resp. $\left.9.9 \pm 1.2 \mathrm{R}_{\odot}\right)$. For the secondary we interpolated the Humphreys \& McElroy temperature scale to obtain values of $T_{\text {eff }}^{\mathrm{Og} .7 \mathrm{~V}}=31.2_{29.8}^{32.6} \mathrm{kK}$. This corresponds to $R_{2}=7.9 \pm 1.7 \mathrm{R}_{\odot}$, where the quoted confidence intervals have been computed by adopting the parameters of the neighbouring spectral sub-types.

\subsection{Masses and orbital inclination}

Fig. 9 presents the location of the stars in the H-R diagram. The evolutionary age of both components is about 4 to $5 \mathrm{Myr}$, which is in agreement with previous cluster age determinations from photometric studies (Perry et al., 1990; Balona \& Lanev, 1995; Raboud et al., 1997; Baume et al., 1999). From a rough interpolation of the tracks, the main sequence primary had an initial mass of about $25 \mathrm{M}_{\odot}$, corresponding to a present mass close to $24.5 \mathrm{M}_{\odot}$, slightly larger than typical O9V masses (which suggests again that the primary component has reached an intermediate stage of evolution between classes V and III). Similarly, the secondary initial mass was about $18.5 \mathrm{M}_{\odot}$. Its present mass should be about $18.2 \mathrm{M}_{\odot}$.
Comparing the obtained minimal mass of Table 4 with typical O9V masses suggests an orbital inclination $i$ of about $70^{\circ}$. Comparing with O9III masses would rather yield $i \sim 60^{\circ}$. Using the constraints deduced on the components radii, we found that the limiting inclination for which eclipses may or not occur in the system lies around $55-65^{\circ}$. Clearly, HD 152218 is a limiting case. Photometric monitoring is required to search for the signature of eclipses or ellipsoidal deformation that could help to constrain the inclination of the system. Finally, we used the formula of Eggleton (1983) to estimate the Roche lobe radii. We found that, according to the previous results, both stars should presently fill about $17 \%$ of their Roche lobe volume.

\section{XMM-Newton observations}

\subsection{Light curves and spectra}

With a typical count rate of about $12 \times 10^{-3} \mathrm{cnt} \mathrm{s}^{-1}$ in the two MOS instruments, HD 152218 is a relatively bright $\mathrm{X}$-ray source. Because the count rates in the hard energy band (2.5-10.0keV) are very low, thus associated with large uncertainties, we restrain our analysis to energies below $2.5 \mathrm{keV}$. Fig. 10 presents the obtained broad band light curves in the total band and in the $\mathrm{S}_{\mathrm{X}}[0.5-1.0 \mathrm{keV}]$ and $\mathrm{M}_{\mathrm{X}}$ [1.0-2.5 keV] bands. Except for Obs. 2 at $\phi \approx 0.7$, the two instruments give very consistent results, indicating a slight modulation with an increase of the X-ray flux of about $30 \%$ at apastron compared to periastron.

To constrain the physical properties of the emitting plasma, we adjusted a series of optically thin thermal plasma (mekal) models using the XSPEC software (Arnaud, 1996). We simultaneously fitted the two MOS spectra of each pointing, adopting an interstellar column of absorbing matter (neutral hydrogen) of $N_{\mathrm{H}, \mathrm{ISM}}=0.29 \times 10^{22} \mathrm{~cm}^{-2}$. This value was obtained using $E(B-V)=0.50$ and the formula of Bohlin et al. (1978). Except for Obs 2 and 4 , for which the effective exposure time was reduced by high background events, better results are obtained using two-temperature (2-T) mekal models. Table 6 summarizes the best-fit values and gives the intrinsic fluxes and luminosities. There is no obvious modulation of the spectral parameters. The modulation of the hardness ratios (Fig. 10) however suggests that the observed emission is slightly harder when the flux level is high, and lower otherwise. The merged spectra are well described by $2-\mathrm{T}$ models with $\mathrm{k} T_{1}=0.31 \mathrm{keV}$ and $\mathrm{k} T_{2}=0.71 \mathrm{keV}$. Fig. 11 displays these spectra together with the best-fit $2-\mathrm{T}$ models. It reveals that a hard emission component $(>5 \mathrm{keV})$ might be present, but clearly the SNR is far insufficient to reliably constrain its properties.

\subsection{HD 152218 wind properties}

We used the constraints on the physical properties deduced in Sect. 4 together with the mass loss recipes of 


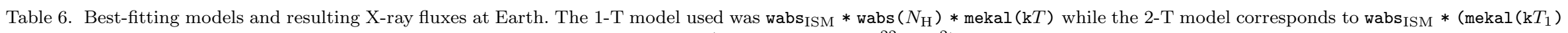

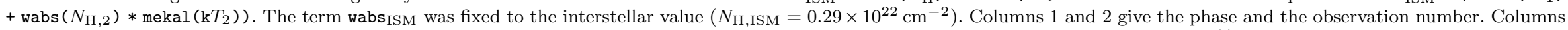

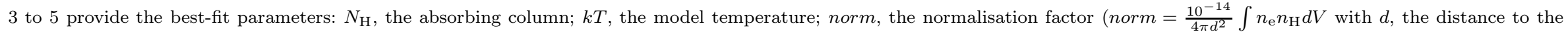

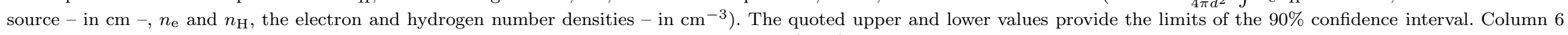

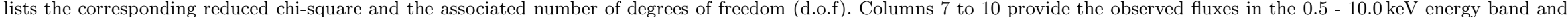

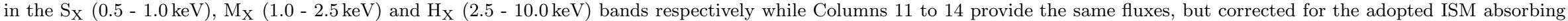
column. Column 15 finally provides the X-ray luminosities in the $0.5-10.0 \mathrm{keV}$ band, assuming $D M=11.07$.

\begin{tabular}{|c|c|c|c|c|c|c|c|c|c|c|c|c|c|c|}
\hline \multicolumn{2}{|c|}{$\phi_{\mathrm{HeI}}$ Obs. \# } & $\begin{array}{c}N_{\mathrm{H}} \\
\left(10^{22} \mathrm{~cm}^{-2}\right)\end{array}$ & \multirow{2}{*}{$\begin{array}{c}\mathrm{k} T \\
(\mathrm{keV}) \\
{[4]} \\
\end{array}$} & \multirow{2}{*}{$\begin{array}{c}\text { norm } \\
\left(\mathrm{cm}^{-5}\right) \\
{[5]} \\
\end{array}$} & \multirow{2}{*}{$\begin{array}{c}\chi_{\nu}^{2} \text { (d.o.f.) } \\
{[6]} \\
\end{array}$} & \multicolumn{3}{|c|}{ 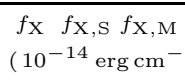 } & $\begin{aligned} f_{\mathrm{X}, \mathrm{H}} \\
\left.2 \mathrm{~s}^{-1}\right)\end{aligned}$ & \multirow{2}{*}{$\begin{array}{c}f_{\mathrm{X}}^{\text {corr. }} \\
(10 \\
{[11]} \\
\end{array}$} & \multicolumn{2}{|c|}{$\begin{array}{l}f_{\mathrm{X}, \mathrm{S}}^{\text {corr. }} f_{\mathrm{X}, \mathrm{M}}^{\text {corr. }} \\
j^{-14} \mathrm{erg} \mathrm{cm}^{-2}\end{array}$} & \multirow{2}{*}{$\begin{array}{r}f_{\mathrm{X}, \mathrm{H}}^{\text {corr. }} \\
\left.\mathrm{s}^{-1}\right) \\
{[14]} \\
\end{array}$} & \multirow{2}{*}{$\begin{array}{c}\log L_{\mathrm{X}} \\
\left(\mathrm{erg} \mathrm{s}^{-1}\right) \\
{[15]} \\
\end{array}$} \\
\hline [1] & {$[2]$} & [3] & & & & [7] & [8] & [9] & [10] & & [12] & [13] & & \\
\hline \multicolumn{15}{|c|}{ Single-temperature (1-T) model } \\
\hline 991 & 1 & $p_{0.02}^{0.19}$ & 0. & 4 & $1.17(47)$ & 8.54 & 4.72 & 3.68 & 0.13 & 20.61 & 14.78 & 5.69 & 0.14 & 1.82 \\
\hline .719 & 2 & $0.11_{0.00}^{0.22}$ & $0.53_{0}^{0}$ & -4 & $0.82(32)$ & 9.275 & 5.07 & 4.05 & 0.15 & 22.20 & 15.80 & 6.24 & 0.16 & 31.85 \\
\hline 873 & 3 & $0.00_{0.00}^{0.03}$ & $0.61_{0.5}^{0.6}$ & $10^{-5}$ & $1.46(47)$ & 8.11 & 4.70 & 3.25 & 0.15 & 19.80 & 14.63 & 5.00 & 0.16 & 31.80 \\
\hline 075 & 4 & $0.38_{0.25}^{0.53}$ & $0.24_{0.19}^{0.31}$ & $0.00 \times 10^{-4}$ & $0.63(35)$ & 7.86 & 4.87 & 2.98 & 0.01 & 22.24 & 17.47 & 4.75 & 0.01 & 1.85 \\
\hline 226 & 5 & $0.40_{0.26}^{0.50}$ & $0.25_{0.2}^{0.3}$ & $10^{-4}$ & 1.35 & 8. & 5.01 & 3.33 & 0.01 & 22.90 & 17.58 & 5.31 & 0.01 & 31.87 \\
\hline 6 & 6 & $0.38_{0.26}^{0.47}$ & $0.24_{0.22}^{0.32}$ & ${ }_{0.00}^{17.3} \times 10^{-4}$ & 0) & 8. & 36 & 3.32 & 0.01 & 47 & 15 & 5.31 & 0.01 & 31.89 \\
\hline & & & & 3.06 & & & & & & & & & & \\
\hline
\end{tabular}




\begin{tabular}{|c|c|c|c|c|c|c|c|c|c|c|c|c|c|c|}
\hline$\overline{\phi_{\mathrm{HeI}}}$ & Obs. \# & $\begin{array}{c}N_{\mathrm{H}} \\
\left(10^{22} \mathrm{~cm}^{-2}\right)\end{array}$ & $\begin{array}{c}\mathrm{k} T \\
(\mathrm{keV})\end{array}$ & $\begin{array}{c}\text { norm } \\
\left(\mathrm{cm}^{-5}\right)\end{array}$ & $\overline{\chi_{\nu}^{2} \text { (d.o.f.) }}$ & $\begin{array}{l}f_{\mathrm{X}} \\
\left(10^{-}\right.\end{array}$ & $\begin{array}{l}f_{\mathrm{X}, \mathrm{S}} \\
-14 \text { er }\end{array}$ & $\begin{array}{l}f_{\mathrm{X}, \mathrm{M}} \\
\mathrm{g} \mathrm{cm}^{-2}\end{array}$ & $\begin{array}{r}f_{\mathrm{X}, \mathrm{H}} \\
\left.2 \mathrm{~s}^{-1}\right)\end{array}$ & 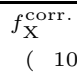 & $\begin{array}{l}f_{\mathrm{X}, \mathrm{S}}^{\text {corr. }} \\
3^{-14} \mathrm{er}\end{array}$ & $\begin{array}{l}f_{\mathrm{X}, \mathrm{M}}^{\text {corr. }} \\
\mathrm{g} \mathrm{cm}^{-}\end{array}$ & $\begin{aligned} f_{\mathrm{X}, \mathrm{H}}^{\text {corr. }} \\
\left.\mathrm{s}_{\mathrm{s}}^{-1}\right)\end{aligned}$ & $\begin{array}{c}\log L_{\mathrm{X}} \\
\left(\mathrm{erg} \mathrm{s}^{-1}\right)\end{array}$ \\
\hline$[1]$ & {$[2]$} & {$[3]$} & {$[4]$} & {$[5]$} & [6] & {$[7]$} & {$[8]$} & {$[9]$} & {$[10]$} & [11] & {$[12]$} & {$[13]$} & {$[14]$} & {$[15]$} \\
\hline \multicolumn{15}{|c|}{ Two-temperature (2-T) model } \\
\hline \multirow[t]{2}{*}{0.591} & 1 & - & $0.34_{0.24}^{0.54} \mathrm{~s}$ & $9.06_{5.59}^{11.3} \times 10^{-5}$ & & & & & & & & & & \\
\hline & & $0.57_{0.24}^{1.13}$ & $0.68_{0.53}^{0.87}$ & $9.97_{5.52}^{16.0} \times 10^{-5}$ & $0.95(45)$ & 9.31 & 5.08 & 3.96 & 0.27 & 24.64 & 18.42 & 5.93 & 0.29 & 31.90 \\
\hline \multirow[t]{2}{*}{0.873} & 3 & - & $0.24_{0.14}^{0.29}$ & $1.11_{0.68}^{2.69} \times 10^{-4}$ & & & & & & & & & & \\
\hline & & $0.10_{0.00}^{0.36}$ & $0.71_{0.61}^{0.83}$ & $5.88_{3.88}^{8.61} \times 10^{-5}$ & $1.07(45)$ & 9.13 & 5.75 & 3.18 & 0.19 & 28.67 & 23.65 & 4.82 & 0.20 & 31.96 \\
\hline \multirow[t]{2}{*}{0.226} & 5 & - & $0.32_{0.24}^{0.41} \varepsilon$ & $8.98_{5.29}^{11.7} \times 10^{-5}$ & & & & & & & & & & \\
\hline & & $0.36_{0.11}^{0.68}$ & $0.71_{0.57}^{0.99}$ & $7.24_{4.22}^{12.0} \times 10^{-5}$ & $1.19(42)$ & 9.12 & 5.22 & 3.67 & 0.24 & 24.84 & 19.05 & 5.54 & 0.25 & 31.90 \\
\hline \multirow[t]{2}{*}{0.396} & 6 & - & $0.31_{0.26}^{0.38}$ & $1.12_{0.88}^{1.36} \times 10^{-4}$ & & & & & & & & & & \\
\hline & & $0.63_{0.37}^{1.00}$ & $0.71_{0.57}^{0.83}$ & $1.06_{0.73}^{1.65} \times 10^{-4}$ & $0.97(48)$ & 9.99 & 5.58 & 4.08 & 0.32 & 27.70 & 21.29 & 6.08 & 0.34 & 31.95 \\
\hline \multirow[t]{2}{*}{-} & Merged & - & $0.31_{0.29}^{0.34} \mathrm{~s}$ & $9.78_{8.74}^{10.8} \times 10^{-5}$ & & & & & & & & & & \\
\hline & & $0.39_{0.28}^{0.50}$ & $0.71_{0.65}^{0.76}$ & $7.83_{6.56}^{9.20} \times 10^{-5}$ & $0.89(164)$ & 9.55 & 5.47 & 3.82 & 0.25 & 26.26 & 20.23 & 5.77 & 0.26 & 31.93 \\
\hline
\end{tabular}


full range: $0.5-10.0 \mathrm{keV}$
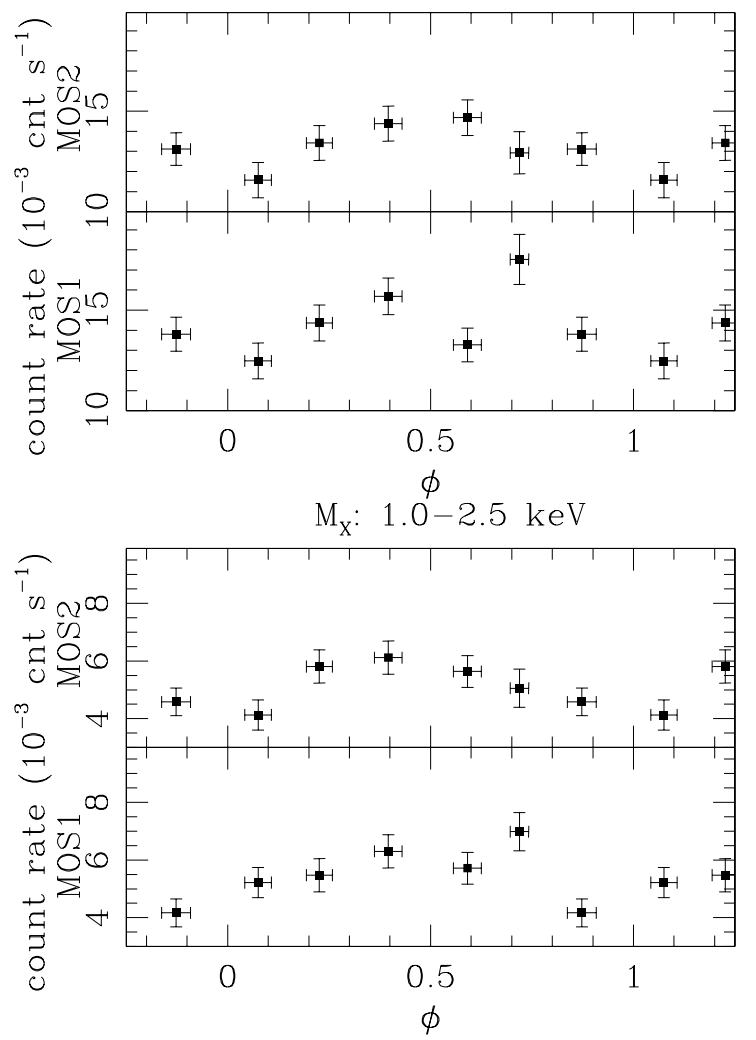
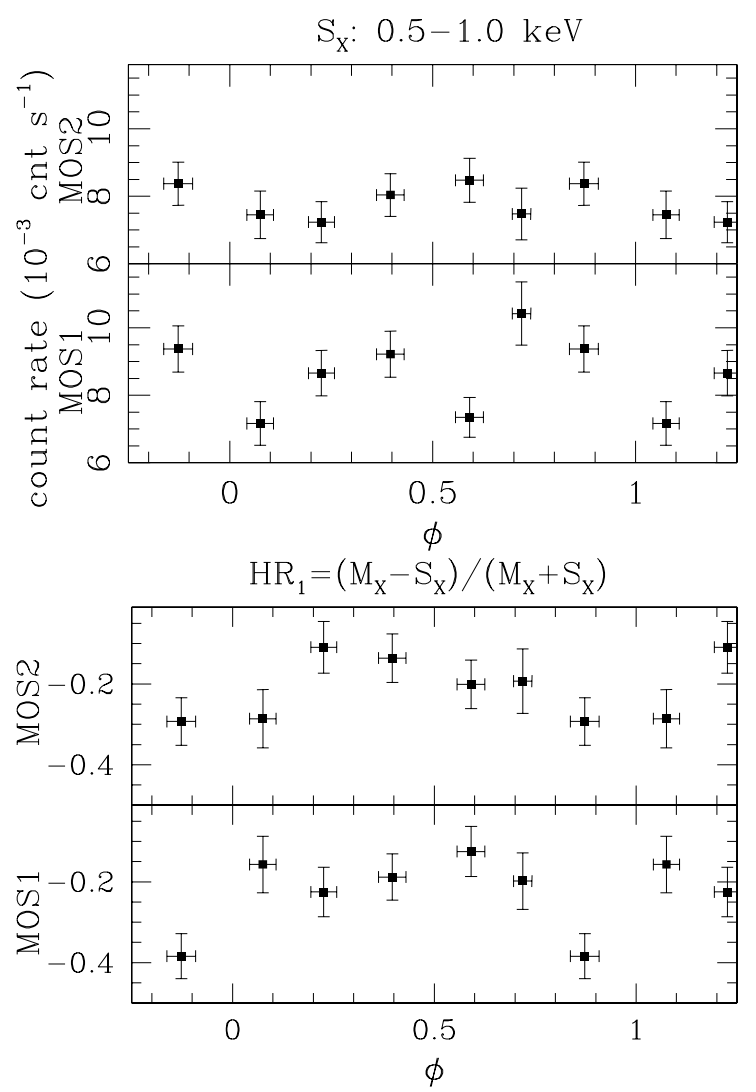

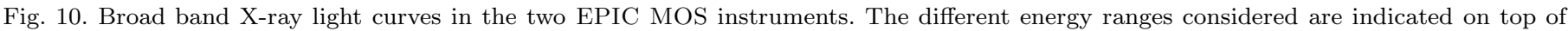

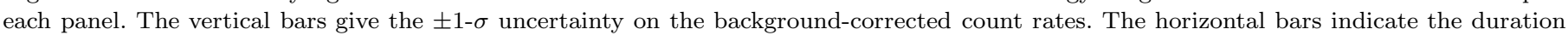
of each pointing. The bottom right panel displays the evolution of the source hardness ratio with the phase, as defined on top of the panel.

Vink et al. (2000, 2001) to estimate the properties of the winds in HD 152218. We obtained $v_{\infty, 1}=2280 \mathrm{~km} \mathrm{~s}^{-1}$, $v_{\infty, 2}=2340 \mathrm{kms}^{-1}, \log \dot{M}_{1}=-6.59\left(\mathrm{M}_{\odot} \mathrm{yr}^{-1}\right)$ and $\log \dot{M}_{2}=-7.37\left(\mathrm{M}_{\odot} \mathrm{yr}^{-1}\right)$. The estimated terminal velocities are only slightly larger than the $2050 \mathrm{~km} \mathrm{~s}^{-1}$ value measured by Howarth et al. (1997). Using these parameters with a $\beta=1$ acceleration law for the winds, we could not establish a ram pressure equilibrium at all phases, indicating that the wind collision structure could be quite unstable. We suspect that the wind velocity law might be strongly affected by radiative inhibition (Stevens \& Pollock, 1994). The latter may even govern the flux of colliding matter. Clearly, only a detailed modeling could allow to get an insight into the wind collision in this system. This is far beyond the goal of the present paper. We will thus limit ourselves to the observational result that the flux is slightly variable and that the maximum of emission is observed near apastron while the minimum is reached at periastron. As HD 152218 is a close binary, the wind interaction will inevitably take place within the acceleration region of the winds. We thus expect the collision to be stronger at apastron, because of the higher pre-shock velocity reached by the wind at this particular phase. However, we caution that more subtle aspects (such as radiative inhibition) probably affect the strength of the winds, and might thus govern the actual position and shape of the on-going wind interaction.

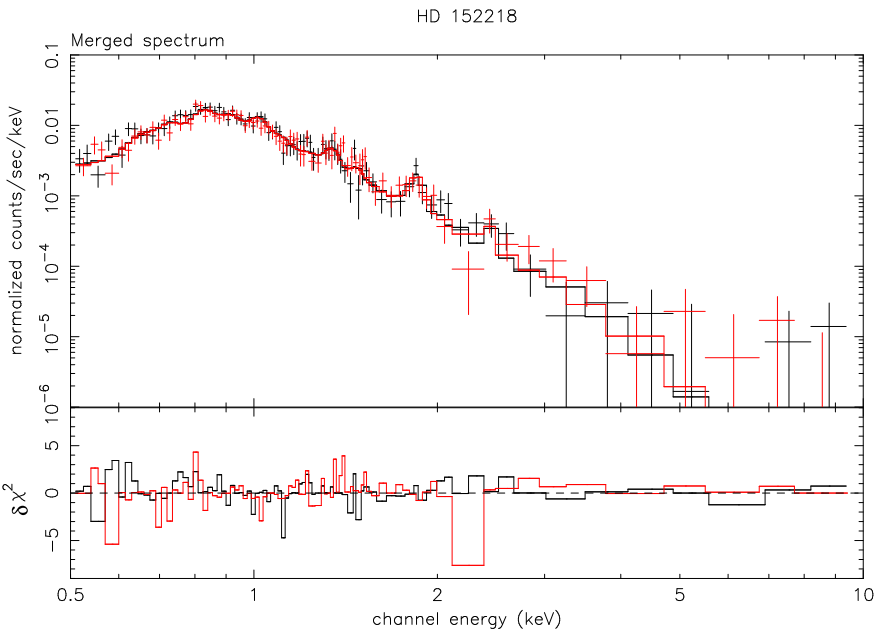

Fig. 11. Top panel: Merged EPIC MOS1 (black) and merged EPIC MOS2 (light grey - hardcopy edition - or red - electronic edition) X-ray spectra of HD 152218 and best-fit $2 \mathrm{~T}$ models. Lower panel: Individual contributions of the different energy bins to the $\chi^{2}$. The contributions are carried over with the sign of the deviation (in the sense data minus model).

\section{Summary}

We have presented the results of a long-term spectroscopic monitoring campaign on the massive binary HD 152218. The last two investigations on the object were pre- 
senting conflicting results. The present data complete the existing observational sets and allow to definitely overcome the ambiguity on the orbital period of the system, bringing to firm ground the period of $5.604 \mathrm{~d}$. From our analysis, we reject the shorter period and higher eccentricity proposed by García \& Mermilliod (2001) and rather confirm the earlier work of Stickland et al. (1997), based on IUE observations. Our newly derived orbital solution is characterized by a slightly lower eccentricity than previously accepted: $e=$ $0.259 \pm 0.006$. The system is most probably formed by an O9 sub-giant and an O9.7 main sequence star. We derived minimal masses of $15.82 \pm 0.26 \mathrm{M}_{\odot}$ and $12.00 \pm 0.19 \mathrm{M}_{\odot}$ and we constrained the component radii to values of $R_{1}=$ $10.1 \pm 1.0 \mathrm{R}_{\odot}$ and $R_{2}=8.9 \pm 2.0 \mathrm{R}_{\odot}$. These values are in good agreement with previous findings and further indicate that HD 152218 should have an orbital inclination of about $60-70^{\circ}$. This corresponds to a limiting case in which eclipses or ellipsoidal variations might be observed.

We also report the results of the monitoring of the system in the X-rays thanks to XMM-Newton observations of the cluster. The averaged X-ray spectrum is relatively soft. It is well reproduced by a $2-\mathrm{T}$ mekal model with component temperatures about 0.3 and $0.7 \mathrm{keV}$. We showed that the system presents an increase of its X-ray flux of about $30 \%$ near apastron compared to periastron. We note that this could be the signature of an ongoing wind-wind interaction process occurring within the wind acceleration region. Such a scenario indeed predicts a stronger shock, and thus a larger X-ray emission from the colliding zone, when the separation between the components is larger, allowing the winds to reach larger pre-shock velocities. This is also supported by the apparent modulation of the hardness ratio, which seems higher when the emission level is stronger. Preliminary computations however indicate that no simple modeling, reproducing the main properties of the interaction, can be obtained. By contrast, the same computations rather point towards second order effects, such as radiative inhibition, to play a major role in this system. These effects could be strong enough to govern the structure and shape of the interaction. HD 152218 might thus be an excellent case to test and/or refine wind collision models, including detailed physics on the wind acceleration and inhibition.

\section{Acknowledgments}

The Liège team is greatly indebted towards the 'Fonds de la Recherche Scientifique' (FNRS), Belgium, for multiple supports. Part of this work was also supported by the PRODEX XMM-OM and Integral Projects, as well as contracts $\mathrm{P} 4 / 05$ and P5/36 'Pôle d'Attraction Interuniversitaire' (Belgium).
Arnaud K. A., 1996, XSPEC: The First Ten Years, in Astronomical Data Analysis Software and Systems V, ASP Conf. Ser. 101, Eds G. Jacoby \& J. Barnes, p. 17

Balona L. A., Laney C. D., 1995, MNRAS, 276, 627

Baume G., Vázquez R. A., Feinstein A., 1999, A\&AS, 137, 233

Bohlin R. C., Savage B. D., Drake J. F., 1978, ApJ, 224, 132

Chlebowski T., Garmany C. D., 1991, ApJ, 368, 241

Conti P. S., 1973a, ApJ, 179, 161

Conti P. S., 1973b, ApJ, 179, 181

Conti P. S., Alschuler W. R., 1971, ApJ, 170, 325

Conti P. S., Leep E. M., Lorre J. J., 1977, ApJ, 214, 759

Eggleton P. P., 1983, ApJ, 268, 368

García B., Mermilliod J. C., 2001, A\&A, 368, 122 (GM01)

Gosset E., Royer P., Rauw G., Manfroid J., Vreux J.-M., 2001, MNRAS, 327, 435

Heck A., Manfroid J., Mersch G., 1985, A\&AS, 59, 63 (HMM85)

Hill G., Crawford D. L., Barnes J. V., 1974, AJ, 79, 1271 (HCB74)

Howarth I. D., Siebert K. W., Hussain G. A. J., Prinja R. K., 1997, MNRAS, 284, 265

Humphreys R. M., McElroy D. B., 1984, ApJ, 284, 565

Jansen F., Lumb D., Altieri B., Clavel J., Ehle M., Erd C., Gabriel C., Guainazzi M., et al., 2001, A\&A, 365, L1

Lafler J., Kinman T. D., 1965, ApJS, 11, 216

Levato H., Morrell N., 1983, Astrophys. Lett., 23, 183 (LM83)

Mason K. O., Breeveld A., Much R., Carter M., Cordova F. A., Cropper M. S., Fordham J., Huckle H., et al., 2001, A\&A, 365, L36

Mathys G., 1988, A\&AS, 76, 427

Mathys G., 1989, A\&AS, 81, 237

Penny L. J., Bagnuolo W. G., Gies D. R., 1994, Sp. Sc. Rev., 66, 323

Perry C. L., Hill G., Younger P. F., Barnes J. V., 1990, A\&AS, 86, 415 (PHYB90)

Raboud D., Cramer N., Bernasconi P. A., 1997, A\&A, 325, 167

Sana H., Hensberge H., Rauw G., Gosset E., 2003, A\&A, 405, 1063

Sana H., Gosset E., Rauw G., 2006a, MNRAS, 371, 67

Sana H., Gosset E., Rauw G., Sung H., Vreux J.-M., 2006b, A\&A, 454, 1047

Sana H., Rauw G., Nazé Y., Gosset E., Vreux J.-M., 2006c, MNRAS, 372, 661

Schaller G., Schaerer D., Meynet G., Maeder A., 1992, A\&AS, 96, 269

Schmidt-Kaler T., 1982, Physical Parameters of the Stars, Landolt-Börnstein, Numerical Data and Functional Relationships in Science and Technology, Vol. 2b, SpringerVerlag, Berlin, pp. 1-34

Stevens I. R., Pollock A. M. T., 1994, MNRAS, 269, 226

Stickland D. J., Lloyd C., Penny L. R., 1997, The Observatory, 117, 213 (SLP97)

Strüder L., Briel U., Dennerl K., Hartmann R., Kendziorra E., Meidinger N., Pfeffermann E., Reppin C., et al. 2001, 
A\&A, 365, L18

Struve O., 1944, ApJ, 100, 189

Sung H., Bessell M. S., Lee S., 1998, AJ, 115, 734

Turner M. J. L., Abbey A., Arnaud M., Balasini M., Barbera M., Belsole E., Bennie P. J., Bernard J. P., et al. 2001, A\&A, 365, L27

Underhill A. B., 1994, ApJ, 420, 869

Vink J. S., de Koter A., Lamers H. J. G. L. M., 2000, A\&A, 362,295

Vink J. S., de Koter A., Lamers H. J. G. L. M., 2001, A\&A, 369,574

Walborn N. R., Fitzpatrick E. L., 1990, PASP, 102, 379

Wolfe R. H., Horak H. G., Storer N. W., 1967, The machine computation of spectroscopic binary elements, in Modern astrophysics. A memorial to Otto Struve, Ed. M. Hack, New-York, Gordon Breach, pp. 251-273 